\title{
Entrelacs
}

ENTRELACS Cinéma et audiovisuel

$14 \mid 2018$

Marchés du film : évolutions, mutations et perspectives

\section{Les festivals et les marchés du film}

Pierre Mazars, Aude Hespert et Hélène Laurichesse

\section{(2) OpenEdition}

Journals

Édition électronique

URL : http://journals.openedition.org/entrelacs/4320

DOI : $10.4000 /$ entrelacs.4320

ISSN : 2261-5482

Éditeur

Éditions Téraèdre

Référence électronique

Pierre Mazars, Aude Hespert et Hélène Laurichesse, "Les festivals et les marchés du film », Entrelacs [En ligne], 14 | 2018, mis en ligne le 03 janvier 2019, consulté le 01 mai 2019. URL : http://

journals.openedition.org/entrelacs/4320 ; DOI : 10.4000/entrelacs.4320

Ce document a été généré automatiquement le 1 mai 2019.

Tous droits réservés 


\title{
Les festivals et les marchés du film
}

\author{
Pierre Mazars, Aude Hespert et Hélène Laurichesse
}

1 Propos recueillis par Hélène Laurichesse, Maître de Conférences à l'ENSAV, Université Toulouse Jean-Jaurès, Chercheuse au LARA-SEPPIA, le 9 décembre 2017 dans le cadre du Colloque «Distribution et ventes internationales des films et séries à l'ère du numérique " organisé par Dominique Bougerol et Kira Kitsopanidou (IRCAV-ICCA, Université Sorbonne Nouvelle Paris 3).

Hèlène Laurichesse : Un certain nombre d'évolutions ont été observées dans le contexte de l'industrie audiovisuelle dans lequel vous exercez votre métier, je pense notamment aux nouveaux modes de diffusion des contenus sur les plateformes, à l'internationalisation croissante des marchés, à l'émergence de nouveaux pays, et à l'importance croissante des coproductions dans le développement des projets. Que pourriez-vous nous dire sur ces sujets au regard de votre expérience?

\section{Les plateformes de streaming}

Aude Hespert : Je peux commencer par évoquer la nouvelle révolution numérique à laquelle nous sommes confrontés avec l'irruption des opérateurs Netflix et Amazon en particulier, avec une arrivée en fanfare à Sundance notamment et Cannes avec des acquisitions à des tarifs faramineux. En 2016, par exemple à Sundance, The Birth of a Nation a été vendu $17 \mathrm{M} \$$, et Netflix avait surenchéri pour $20 \mathrm{M} \$$... on atteint des records. Manchester by the sea a été acquis par Amazon pour 10 M\$. Cette année encore Netflix a été hyper puissant, il a acheté neuf titres. Il y a eu l'effet Sundance puis ensuite l'affaire Netflix à Cannes avec non seulement des films produits par Netflix, mais aussi si on remonte à 2016, trois films français avaient été achetés pour des sorties «monde » directement en VOD. Le film de Houda Benyamina Divines, qui a eu une grande notoriété car il a eu la caméra d'or, avait été acheté avant d'avoir le prix et les deux autres films c'était Le voyage au Groenland de Sébastien Betbeder et Mercenaire de Sacha Woff. Des films dont peut-être vous n'avez même pas entendu parler. Dans tous les cas évidemment il y a beaucoup de questionnements par rapport à ces nouveaux enjeux. Est-ce que des distributeurs indépendants pourront s'aligner sur les tarifs proposés par ces nouveaux 
opérateurs? Et puis comment continuer à promouvoir un auteur qui va voir ses films directement diffusés en VOD surtout quand il n'a pas encore de notoriété internationale. Par exemple, lorsque le film de Bertrand Bonello est acheté par Netflix, on imagine qu'il va être vu parce qu'il s'agit d'un auteur déjà reconnu, mais pour les autres auteurs il y a cette réflexion-là qui est importante aujourd'hui à Unifrance sur la promotion des auteurs et des films français.

Pierre Mazars : Dans ma structure de vente Charades, on est aussi bien coproducteurs que vendeurs internationaux. Je pense que d'une certaine manière, le métier de vendeur est en train de disparaître, on se positionne davantage comme des financiers à l'international que comme vendeurs même si la vente est au cœur de notre activité. Mon sentiment par rapport au phénomène, à la rupture à laquelle on est en train d'assister avec l'arrivée de ces nouveaux intervenants, c'est qu'on est en train de vivre une bulle spéculative qui est amenée à disparaître dans les années à venir. Netflix, c'est des gens qui ont énormément d'argent, mais qui ne publient quasiment aucun de leurs comptes. Je pense qu'ils perdent beaucoup d'argent étant donné que leur seul modèle économique, c'est cette structure de distribution. C'est une sorte de géant aux pieds d'argile. Les phénomènes d'achats qui ont été très présents à Cannes et à Sundance l'année dernière sont déjà en train de ralentir. En même temps, je vous dis ça alors que je viens de vendre à Netflix un film qui s'appelle Revenge il y a quelques mois. Ce film avait été pris à Sundance, il était aussi Toronto en avant-première et on l'a vendu à Netflix pour les territoires restants, c'est-à-dire qu'on l'a vendu en Asie du Sud-Est où on n'arrivait pas à trouver de distributeurs. Autrement dit, on a fini notre exploitation avec Netflix.

tier d'exportateur de films est une spécificité française, il y a quarante structures d'exportations en France, on est les premiers au monde, on a une expertise reconnue sur l'exportation nous les Français, et je pense qu'on doit continuer à se battre pour travailler sur des modèles vertueux qui permettent de continuer l'exploitation en salles de cinéma. Les exploitants ont tendance à beaucoup râler à propos de Netflix, ils disent que les salles de cinéma vont toutes fermer... Ils disaient la même chose quand il y a eu l'ORTF dans les années 50, ils ont dit pareil à propos de la VHS et du DVD .... Mais les salles de cinéma continueront à exister si on continu tous à considérer le cinéma comme le premier loisir public sur la planète.

6 Netflix, c'est juste un phénomène qui va se tasser de lui-même, il ne faut ni le considérer comme un eldorado ni s'affoler. Amazon, c'est autre chose, ils peuvent investir dans le cinéma autant qu'ils veulent, ils vendent des millions de machines à laver et de vêtements. Amazon n'est pas un colosse aux pieds d'argile, l'audiovisuel pour eux c'est un petit business, une vitrine, sur une énorme masse financière qui dépasse l'entendement. Ils peuvent s'amuser à claquer autant de pognon qu'ils veulent tellement ils en gagnent à côté. Pour eux le risque n'est pas fort et d'ailleurs quand Amazon achète des films, ils les sortent en salles contrairement à Netflix. Amazon trouve des deals de distribution. Le vrai modèle, il est là, je pense que les intervenants vont changer. Avant je vendais mes films à Weinstein, qui est en train de s'effondrer pour d'autres raisons, maintenant je vais vendre mes films à des plateformes VOD (AMC par exemple qui ne fait que des films de genre dans les pays anglophones). Avant on entrait par la salle de cinéma, maintenant on va rentrer par les networks et les métiers de la distribution digitale, mais on peut penser que le métier de l'exploitation en salle va continuer, c'est l'enjeu et le combat qu'on doit mener ensemble sur nos métiers. 


\section{La coproduction}

7 Pierre Mazars : Avant, quand un producteur français voulait financer un film, il allait voir Canal Plus, TF1 et M6. Maintenant ce qu'on appelle la free TV - les chaînes hertziennes - ont baissé drastiquement leurs investissements, car ils préfèrent diffuser les experts et autres séries TV. Le cinéma a beaucoup pâti de ça, et la nouvelle génération de producteurs a compris que les sources de revenus se faisaient maintenant à l'international. Donc pour cela, ils vont voir les vendeurs qui disent : dans ce cas, si vous avez besoin de nous, en échange, on va rentrer dans la coproduction et on va vous aider à internationaliser votre film.

Aude Hespert : Chez Unifrance et au CNC aussi c'est un enjeu très important les coproductions, pas seulement pour le montage financier du film, mais aussi pour leur diffusion ultérieure, car on se dit que s'il y a plusieurs pays impliqués, chacun sera à même de trouver le meilleur distributeur sur les zones concernées, donc c'est aussi dans un souci que le film soit vu à l'international au maximum.

9 Pierre Mazars : Le concept de film franco-français est amené à disparaître. Enfin, ça peut exister pour les Tuches et ce cinéma-là, et c'est une bonne chose, car ça fait vivre une partie de l'économie, mais ce n'est pas notre domaine d'action. Le cinéma français est en train de muter, c'est-à-dire que les films sont européens, internationaux, ils parlent en anglais, en français et tout le monde s'en fiche, la jeune génération s'en fiche, ils passent de l'un à l'autre instinctivement. Rentrer en coproduction, c'est contribuer à cette internationalisation du cinéma et à la rencontre des talents. Quand on produit un film maintenant on fait appel à des chefs opérateurs espagnols, des comédiens italiens et ça permet d'avoir un maximum de talents pour le projet. Donc, c'est plutôt une bonne chose, et la plupart des vendeurs petit à petit comprennent qu'il faut entrer comme coproducteur, et ça veut dire avoir des moyens financiers plus importants. Mais comme les banques ont envie d'investir dans le cinéma - ça, ne me demandez pas pourquoi, car ils perdent de l'argent, je pense - c'est le bon moment pour aller sur cette tendance. Quand Valerian sort dans le monde entier, personne ne sait que c'est français, tout le monde s'en fiche, or, c'est un film $100 \%$ français. Le concept de nationalité maintenant est complètement flou et tant mieux pour l'économie globale.

\section{Chronologie des médias}

10 Pierre Mazars : Elle a tendance à disparaître et c'est tant mieux. Dans les autres pays où elle n'est pas et où chacun fait ce qu'il veut, ça n'est pas un problème, ça ne pénalise pas les salles. Il y a eu une conférence du patron de Netflix - je ne suis pas toujours d'accord avec ce qu'il dit, mais là il a eu un exemple pas mal - il a dit: Imaginez si les hypermarchés imposaient au consommateur de ne pas aller au restaurant pendant trois mois pour aller faire des courses et manger chez eux. Tout le monde trouverait ça aberrant et pourtant c'est ce qui se passe au cinéma, on vous interdit de voir un film pendant quatre mois chez vous pour vous obliger à le voir au cinéma. C'est complètement invraisemblable comme système. On a envie de voir un film au cinéma parce que c'est une expérience collective et on le regarde chez soi parce qu'on n'a pas envie de sortir, c'est une autre expérience. Espérons qu'un jour ces débats autour de la chronologie des médias 
s'arrêtent enfin et que les gens qui font du business puissent faire au mieux pour les films et que les gens qui les regardent puissent les regarder comme ils le veulent.

\section{Vendeurs versus financiers internationaux}

11 Pierre Mazars: Prenons le cas pratique du film Revenge qui coûte $2 \mathrm{M€}$. Avec mes associés, on évalue la valeur internationale du film et on décide de mettre $200000 €$. À quinze jours du tournage, le producteur nous dit «Je suis désolé, il me manque $400000 €$, je n'ai pas eu l'aide bancaire que j'espérais, toute la prépa est faite, les acteurs sont là, mais le film ne va pas se faire. On va arrêter le projet ». Donc, on a rajouté $400000 €$ pour arriver à 600000 et on est entré dans le projet en tant que financier. Ces $6000000 €$ bien sûr, ils ne sont pas à nous, on fait appel à un fonds spécialisé dans le cinéma qui aide les films à se monter au dernier moment. En échange de ce risque, on prend $20 \%$ de coproduction du film, donc on a notre mot à dire sur le casting, les premières images qu'on voit, le montage, tous les éléments qui vont constituer le film. Alors qu'en tant que vendeur, on n'avait pas notre mot à dire. Il est de plus en plus courant aujourd'hui que les producteurs s'aperçoivent que les banques ou les chaînes ne les suivent plus, c'est pourquoi on est devenu un guichet de plus en plus important dans le financement des films. Pour cela, soit on apporte cet argent nous-mêmes, soit on fait appel à ces fonds privés. Maintenant on va aussi pouvoir faire appel aux SOFICA puisqu'elles vont avoir une obligation d'investir dans l'exportation de films, donc elles vont venir nous voir pour qu'on dépense leur argent.

\section{L'importance respective des marchés}

12 Pierre Mazars: Les trois marchés les plus importants sont Berlin Cannes et l'AFM (American Film Market). Aujourd'hui, Cannes a un poids écrasant, cela devient un problème, car si vous n'êtes pas bien représenté à Cannes, votre année est foutue. Vous jouez votre année sur quinze jours aussi bien en termes de business, que d'image que de promotion de vos films. Il faudrait que les autres festivals aient plus de répercussions. Quel film a gagné Venise par exemple? Très peu le savent même parmi les professionnels.

Aude Herspert : On s'amuse à Unifrance à comptabiliser les films qui voyagent le plus et ce sont systématiquement ceux qui étaient présents à Cannes. Cannes est devenu un entonnoir, les vendeurs attendent Cannes pour présenter un film. Berlin est plutôt en retrait dans la stratégie des vendeurs.

Pierre Mazars : On utilise Berlin pour mettre la pression à Cannes, on montre à Berlin en espérant qu'ils aiment bien et on va dire non pour avoir le film à Cannes, voilà c'est à cela que sert Berlin maintenant.

Aude Hespert : Après, il y a des marchés alternatifs intéressants, avoir un film français en compétition à Sundance par exemple ça peut être extraordinaire, on peut avoir toute la presse américaine et ça peut être mieux que d'être à Berlin. Le travail des vendeurs, c'est justement d'avoir une stratégie festival par rapport au film, de trouver le meilleur endroit pour le présenter et ne pas jouer uniquement la carte de Cannes.

Pierre Mazars : Cannes est toujours la plus grande vitrine, Berlin a, je pense, à une carte à jouer sur le plan européen, l'AFM à Los Angeles est en train de décliner, inversement, 
Toronto qui est un faux marché (il n'y a pas de lieux pour acheteurs et vendeurs) est en train de progresser.

\section{Les coulisses d'un marché}

17 Pierre Mazars : Notre métier de vendeur c'est comme un métier de sportif, on passe son temps à s'entraîner chez soi dix mois par an et ensuite pendant deux mois on a des compéts et il faut assurer, et là on bosse dix-huit heures par jour. Donc il faut se préparer, cela signifie avoir du lobbying pour essayer d'avoir ses films dans les festivals avec par exemple le concours d'Unifrance. Cela veut dire aussi convaincre les producteurs d'avoir les meilleurs outils de vente, ensuite c'est un métier qui se passe au relationnel et à la connaissance du marché avec ses clients, c'est un métier qui fonctionne énormément à la confiance. C'est très important d'avoir une image de marque qu'on réussit à travailler au fil du temps, une ligne éditoriale qui fait que les clients (les distributeurs) vous font confiance sur le film qu'ils vont vous confier. Mais le processus de travail dépend des profils des acheteurs. Certains clients au Moyen-Orient, qui ont beaucoup d'argent arrivent et disent « on prend tout ", il n'ont rien vu, ne connaissent pas les films, mais ils veulent tout prendre. Pareil pour des clients chinois, c'est le Far Ouest, le prochain eldorado sera l'Afrique. Ils achètent les films, ne les voient pas et ils ne les sortent jamais. Pour d'autres pays en revanche ça prend beaucoup de temps on envoie le line up en première étape. À partir de là, ils lisent le scénario, ils trouvent ça nul, veulent discuter, ils parlent du budget du film, ils trouvent que l'actrice n'est pas la bonne et ça va prendre neuf mois de discussion de faire tout ça.

Dans tous les cas, pendant toute cette période, on vend des films qui n'existent pas, on vend du contrat, une promesse c'est pourquoi la notion de confiance est très importante, pour les clients traditionnels en tout cas.

19 Aude Hespert: Concernant les modes de distribution, le numérique est un mode alternatif pour des films difficiles à vendre. Les distributeurs ont toujours l'espoir de vendre à une salle et attendent avant de vendre à une plateforme qui va acheter le film pas très cher. Je pourrais vous parler d'une initiative qu'on a lancée à Unifrance, « Young French cinéma ». Ce sont des films français qui n'ont pas de distributeur américain dont on fait la promotion lors d'un congrès d'exploitants. On le propose à toutes les salles arts et essai et sur les campus américains qui ont souvent des salles de cinéma. Grâce à cette initiative, on peut faire circuler les films alors que ceux qui ont un distributeur classique ne sortent qu'à Los Angeles et New York. Ça marche plutôt bien, les distributeurs s'y retrouvent, car il y a une remontée de recettes. Alors, on essaye d'ailleurs de décliner ce modèle sur d'autres territoires, comme au Canada. Il y a Amazon aussi qui depuis deux ans propose dans certains festivals comme Toronto d'acheter les films qui n'auraient pas trouvé de distributeur. Mais cela reste une solution de secours.

Pierre Mazars : Les festivals ne sont pas à négliger en effet. Pour nous, c'est une source de business alternatif.

\section{Différence entre contrat de distribution et mandat de distribution}

21 Pierre Mazars : Dans un mandat de distribution simple, on ne verse pas de minimum garanti, c'est une manière d'acquérir les droits, c'est un cas assez rare maintenant, car de 
plus en plus on entre dans la production du film et on a une avance à verser. On joue sur les deux volets en tant que vendeur et en tant de producteur. Quand on peut, on essaye de demander la loi française, on essaye de se battre pour négocier ça, mais ça dépend du territoire en question, notre line-up est consituté à seulement $20 \%$ de films français, on doit parfois transiger avec les lois en vigueur dans les autres pays. Quand on travaille avec des Russes, on se bagarre pour que ce soit la loi française. Avec Netflix, c'est un rapport de force, on ferme juste sa gueule, on ne peut rien négocier. Dans notre modèle on se rembourse des frais... les producteurs pensent toujours qu'on va les arnaquer, quand on va à Cannes, on se fait rembourser les frais puisqu'on a des bureaux, des billets d'avion, etc... on se les rembourse sur les recettes du film. Ces frais sont plafonnés entre 50000 et $150000 €$, mais c'est souvent un débat avec les producteurs qui pensent qu'on va passer des vacances avec. Ce sont peut-être des choses qui sont arrivées dans le passé, mais maintenant ce n'est plus le cas, car l'économie est tellement serrée au niveau des contraintes de financement que on peut plus se permettre ce genre de choses, mais c'est un énorme débat.

\section{Paiement des producteurs}

Pierre Mazars : Pour les payer, on fait les recettes moins notre commission - entre 15 et $30 \%-$ moins les frais, moins l'avance qu'on a versée et après on reverse au producteur. C'est la méthode la plus simple, mais finalement je n'applique ce modèle sur aucun de mes films, car soit il y a une Sofica, soit un fonds privé à rembourser. Il faut une grande souplesse aujourd'hui pour faire de la gymnastique financière, et ce modèle est quasi terminé et tant mieux, car ce qui est le plus excitant dans nos métiers, c'est de trouver les solutions pour que les films puissent se faire. Pour prendre l'exemple d'un documentaire sur le reggae qu'on est en train de produire, je touche $20 \%$ jusqu'à $250000 €$ de recettes, entre 250000 et $600000 €$, je ne touche rien, à partir de $600000 €$ je récupère ma quotepart sur les $20 \%$ entre 250000 et 600000 , plus un bonus de $5 \%$ au-dessus de $750000 €$. Cela permet au film de se faire, il a son couloir de recettes réservé entre 250 et 600 000 qu'il peut ajouter en trésorerie dans le film. Tous les systèmes traditionnels sont en train d'éclater et c'est plutôt marrant. Moi je trouve ça marrant en tout cas.

\section{Les marchés en développement}

\section{L'Afrique}

Pierre Mazars : Le dernier eldorado comme je le disais c'est le marché africain, pour l'instant c'est un marché encore embryonnaire. Des salles se construisent, des distributeurs commencent à s'installer sur place, notamment au Mali. D'un point de vue business, cela ne représente encore rien. Mais je pense que ça va se mettre en place progressivement. Il y a un marché local qui existe, Le Nigeria est quand même le troisième plus gros producteur de films au monde. Les studios américains sont déjà installés, ça va prendre du temps mais je pense que dans vingt ans ce sera une économie comparable à celle qu'on observe aujourd'hui dans les pays d'Asie au niveau du développement. Mais d'ici là, il y a tout un cadre à mettre en place, il y a le piratage contre lequel il faut lutter, il y a la relation avec les sociétés de télécommunications, dans quelles mesures eux veulent aller vers le marché salles ou plutôt VOD. 
24 Aude Hespert : Ce n'est pas simple, l'offre n'est pas toujours adaptée au marché, la place de cinéma est très chère, c'est plutôt élitiste. Par ailleurs, la France a aidé un certain nombre de films pour la numérisation, avec le cahier des charges de diffuser des films français, mais à l'arrivée ce sont pourtant des films américains qui y sont montrés.

Pierre Mazars : Pour l'instant c'est un marché à perte, mais cela va évoluer, je suis plutôt confiant sur l'avenir.

\section{La Chine}

Pierre Mazars : La chine, c'est le Far Ouest .. Il y a a peu près maintenant soixante, soixante-dix structures de distribution. Une structure de distribution se monte toutes les deux heures avec des noms aussi exotiques que Mr Rikiki, Mr Ramolo. Ils vous disent: "Votre film j'ai très envie de l'acheter, j'ai une structure de distribution énorme en Chine ». Moi je n'en ai jamais entendu parler, personne ne la connait, mais c'est une structure qui a levée des millions. On a eu douze structures dans la journée qu'on ne connaissait pas qui nous ont proposé un million de dollars et demi pour le film d'animation qu'on avait à la vente.

27 Aude Hespert : En fait, à Cannes ce qui s'est passé, ce que nous ont rapporté les exportateurs d'Unifrance, c'est qu'il y a eu de nombreuses ventes de catalogue à des acheteurs chinois, mais ils ne vont jamais sortir ces films, ils les achètent pour valoriser en bourse leur catalogue, c'est comme ça que ça se passe.

Pierre Mazars : C'est une bulle spéculative, ils ne veulent pas distribuer les films en fait, il y a quatre ou cinq vrais distributeurs en Chine, ils spéculent pour acheter le film et le revendre après. Ce qu'ils essayent de faire passer c'est les clauses de censure, mais nous on les refuse, car si le film ne passe pas la censure, il faudrait les rembourser.

Aude Hespert : Sept films français par an sont acceptés par la censure, mais on n'arrive pas à trouver sept films par an qui arrivent à passer la censure, donc on ne souhaite pas vraiment augmenter ce nombre. Mais lorsqu'on passe la censure, là c'est bingo car il y a tellement de salles...

Pierre Mazars : Alors à l'arrivée, on a eu toutes ces offres à 1,5 M\$, mais on l'a vendu à 1,2 car on a préféré privilégier une structure de distribution établie, qui est une vraie structure avec de vraies gens plutôt que d'aller vers des structures où on ne sait pas ce qui va se passer avec les films.

31 Aude Hespert : Il est question d'ouvrir des salles art et essai en Chine où on pourrait montrer des films en dehors de la censure, mais pour le moment on attend. Le cinéma chinois a besoin de contenu, et actuellent le marché est très faible, les Chinois produisent pour le marché local, mais ne parviennent pas à le faire au niveau international.

Pierre Mazars : Ils vont s'exporter un jour, c'est certain, le premier coproducteur international qui va réussir à produire un film suffisamment commercial, il va se faire une fortune. On se dit qu'il y aura un film un jour qui va cartonner. On nous propose des projets, des films de science-fiction à $100 \mathrm{M} \$$ de budget. Les producteurs chinois veulent travailler avec tout le monde, même avec nous, on n'a pas les moyens, mais ils nous contactent quand même. La France est un territoire mythique pour les Chinois, il suffit de voir Boulevard Haussmann à Paris tous les Chinois devant les Galeries Lafayette à $8 \mathrm{~h}$ le matin qui font la queue pour entrer pour comprendre l'intérêt. Il y a maintenant des magasins qui sont juste pour les Chinois, où tout est en chinois. Entre l'attrait historique 
du cinéma français et la puissance des Chinois, les passerelles vont être de plus en plus fortes. Les obstacles sont pour le moment politiques, mais ça va s'assouplir.

\section{AUTEURS}

\section{PIERRE MAZARS}

Pierre Mazars est Directeur Général de la société Charades (ventes internationales, exportation, distribution).

\section{AUDE HESPERT}

Aude Hespert occupait au moment de l'entretien le poste de Directrice du service Festival à Unifrance. Elle est aujourd'hui Directrice Générale Adjointe de la société Le Public Système Cinéma

\section{HÉLÈNE LAURICHESSE}

Hélène Laurichesse est Maître de Conférences à l'ENSAV (Ecole Nationale Supérieure d'Audiovisuel) de l'Université Toulouse-Jean-Jaurès et chercheuse au LARA-SEPPIA. Ses recherches portent sur le fonctionnement de l'industrie cinématographique à travers les pratiques des différents acteurs de la filière (auteurs, réalisateurs, producteurs, distributeurs, exploitants) et les stratégies qui s'y déploient. 